\title{
MAXIMAL SUBGROUPS OF INFINITE DIMENSIONAL GENERAL LINEAR GROUPS
}

\author{
DUGALD MACPHERSON
}

(Received 29 November 1990; revised 4 April 1991)

Communicated by $\mathbf{H}$. Lausch

\begin{abstract}
Let $\kappa$ be an infinite cardinal, $F$ a field, and let $\mathrm{GL}(\kappa, F)$ be the group of all non-singular linear transformations on a $\kappa$-dimensional vector space $V$ over $F$. Various examples are given of maximal subgroups of $\mathrm{GL}(\kappa, F)$. These include

(i) stabilizers of families of subspaces of $V$ which are like filters or ideals on a set,

(ii) almost stabilizers of certain subspaces of $V$,

(iii) almost stabilizers of a direct decomposition of $V$ into two $\kappa$-dimensional subspaces. It is also noted that $\mathrm{GL}(\kappa, F)$ is not the union of any chain of length $\kappa$ of proper subgroups.
\end{abstract}

1991 Mathematics subject classification (Amer. Math. Soc.): 20 B 07.

\section{Introduction}

Throughout this paper $\kappa$ will denote an infinite cardinal, $F$ a field, $V$ a vector space of dimension $\kappa$ over $F$, and $G:=\mathrm{GL}(\kappa, F)$ will be the group of all non-singular linear transformations from $V$ onto itself. This paper is mainly concerned with maximal subgroups of $G$, and its goal is to extend to $G$ certain results from [1], [7] and [8] on maximal subgroups of infinite symmetric groups. The examples given of maximal subgroups are very far from exhaustive, and there are several further natural candidates for maximal subgroups which remain to be checked. Also, it would be natural to extend the results further to infinite dimensional classical groups preserving a form. I have not attempted this.

In Section 2 we define notions of filter and ideal on the lattice of subspaces

(C) 1992 Australian Mathematical Society 0263-6115/92 \$A2.00+0.00 
of $V$. The main theorem (Theorem 2.3) of this paper is that stabilisers in $G$ of maximal filters or ideals are maximal in $G$. In fact, the action induced by $G$ on any orbit of maximal ideals or filters is 2-transitive. The proof is straightforward. It is similar to the result of Richmann [9] that if $S$ is the symmetric group on an infinite set $\Omega$ then the stabiliser in $S$ of a maximal filter or ideal on $\Omega$ is maximal in $S$. We also note, in Theorem 2.12 , that, in an appropriate sense, the almost stabiliser of any subspace of $V$ is maximal in $G$. Another maximal subgroup of $G$, given in Theorem 2.13 , is the almost stabiliser of a direct sum decomposition of $V$ into two $\kappa$-dimensional subspaces. In Section 3 we add some miscellaneous remarks about $G$. In particular, we note in Theorem 3.1 that $G$ is not the union of a $\kappa$-chain of proper subgroups (this is similar to a result from [8] about symmetric groups).

It is convenient to recall here the classification of the normal subgroups of $G$, due to Rosenberg [10]. Let $F^{*}$ denote the multiplicative group of $F$. For each infinite cardinal $\lambda \leq \kappa$, let

$$
G_{\lambda}:=\{g \in G: \operatorname{Dim}(V / \operatorname{Fix}(g))<\lambda\}
$$

(here $\operatorname{Fix}(g)$ is the subspace of $V$ consisting of vectors fixed by $g$ ). Note that $g \in G_{\lambda}$ if and only if $\operatorname{Dim}(\operatorname{Im}(g-1))<\lambda$. Then the normal subgroups of $G$ which are not contained in $G_{\omega}$ are precisely the groups of the form $G_{\lambda} \cdot Z$, where $Z \leq F^{*}$. We put $G_{\lambda}^{*}:=G_{\lambda} \cdot F^{*}$. The normal subgroups of $G$ which are contained in $G_{\omega}$ are also described in [10], and were classified by Dieudonné in [2] (they are the natural analogues of the normal subgroups of $\mathrm{GL}(n, F))$.

We will use the following standard permutation group notation. If $H$ is a permutation group on a set $Y$, and $A \subseteq Y$, then $H_{\{A\}}, H_{(A)}$ denote respectively the setwise and pointwise stabilisers of $A$ in $H$.

Several people have made helpful contributions to this paper through conversation. In particular, I mention Paul Bankston, Claudia Böttinger and Wilfrid Hodges.

\section{Maximal subgroups of $\operatorname{GL}(\kappa, F)$}

First, we record the following lemma of Peter Neumann, given in the present form in [8].

Lemma 2.1 [8, Lemma 2.1]. If $\Omega$ is an infinite set, $S:=\operatorname{Sym}(\Omega)$, and $\Gamma, \Delta$ are subsets of $\Omega$ with $|\Gamma \cap \Delta|=\operatorname{Min}\{|\Gamma|,|\Delta|\}$, then

$$
S_{(\Omega \backslash(\Gamma \cup \Delta))}=\left\langle S_{(\Omega \backslash \Gamma)}, s_{(\Omega \backslash \Delta)}\right\rangle \text {. }
$$


This lemma has the following linear analogue, which we use repeatedly. If $U \leq W \leq V$ we say that $U$ is a moietous subspace of $W$ if $\operatorname{Dim} U=$ $\operatorname{Dim} W=\operatorname{Dim} W / U$.

Proposition 2.2. Let $V$ be the direct sum of moietous subspaces $U_{1}, U_{2}$, $W$, and put $H_{1}:=G_{\left(U_{2}\right),\left\{W+U_{1}\right\}}, H_{2}:=G_{\left(U_{1}\right),\left\{W+U_{2}\right\}}$. Then $G=\left\langle H_{1}, H_{2}\right\}$.

Proof. Let $\mathscr{B}_{1}=\left(u_{1 \lambda}: \lambda<\kappa\right), \mathscr{B}_{2}=\left(u_{2 \lambda}: \lambda<\kappa\right)$ and $\mathscr{B}_{W}=\left(w_{\lambda}:\right.$ $\lambda<\kappa)$ be ordered bases for $U_{1}, U_{2}$, and $W$ respectively, and let $\mathscr{B}$ be the basis $\mathscr{B}_{1} \cup \mathscr{B}_{2} \cup \mathscr{B}_{W}$ of $V$. Note that by Lemma $2.1,\left\langle H_{1}, H_{2}\right\rangle$ induces the full symmetric group on $\mathscr{B}$, so we need only show it is transitive on the set of ordered bases of $V$. Let $\mathscr{B}^{\prime}$ be a basis of $V$. Also let $\pi_{1}, \pi_{2}$ and $\pi_{W}$ be the natural projections of $V$ onto $U_{1}, U_{2}$ and $W$.

Claim 1. There is $h_{1} \in\left\langle H_{1}, H_{2}\right\rangle$ such that $\mathscr{B}^{\prime} h_{1} \supseteq \mathscr{B}_{1}$.

Proof of Claim. First, since $\left\langle\mathscr{B}^{\prime} \pi_{1}\right\rangle=U_{1}$ there is $k_{1} \in H_{1}$ such that $\mathscr{B}^{\prime} k_{1} \pi_{1} \supseteq \mathscr{B}_{1}$. For each $\lambda<\kappa$ choose $x_{\lambda} \in \mathscr{B}^{\prime} k_{1}$ such that $x_{\lambda} \pi_{1}=u_{1 \lambda}$, and write $x_{\lambda}=u_{1 \lambda}+v_{\lambda}$ where $v_{\lambda} \in W \oplus U_{2}$. There is a moiety $A$ of $\kappa$ such that $\left\langle v_{\lambda}: \lambda \in A\right\rangle$ has codimension $\kappa$ in $W \oplus U_{2}$. Choose $k_{2} \in\left\langle H_{1}, H_{2}\right\rangle$ so that $\left\{u_{1 \lambda}, v_{\lambda}: \lambda \in A\right\} k_{2}$ spans a moietous subspace of $U_{1}$. Then choose $k_{3} \in H_{1}$ so that $x_{\lambda} k_{2} k_{3}=u_{1 \lambda}$ for all $\lambda \in A$. Finally, choose $k_{4} \in\left\langle H_{1}, H_{2}\right\rangle$ so that $\left\{u_{1 \lambda}: \lambda \in A\right\} k_{4}=\left\{u_{1 \lambda}: \lambda<\kappa\right\}$, and put $h_{1}=k_{1} k_{2} k_{3} k_{4}$. This proves the claim.

Next, since $\left\langle H_{1}, H_{2}\right\rangle$ induces the symmetric group on $\mathscr{B}$ there is $h_{2} \epsilon$ $\left\langle H_{1}, H_{2}\right\rangle$ such that $\mathscr{B}^{\prime} h_{1} h_{2} \supseteq \mathscr{B}_{1} \cup \mathscr{B}_{W}$. Thus,

$$
\mathscr{B}^{\prime} h_{1} h_{2}=\mathscr{B}_{1} \cup \mathscr{B}_{W} \cup\left\{\sum_{\mu<\kappa} a_{\lambda \mu} u_{1 \mu}+\sum_{\mu<\kappa} b_{\lambda \mu} w_{\mu}+u_{\lambda}^{\prime}: \lambda<\kappa\right\}
$$

where $\left(u_{\lambda}^{\prime}: \lambda<\kappa\right)$ is a basis for $U_{2}$, and $a_{\lambda \mu}, b_{\lambda \mu} \in F$ for all $\lambda, \mu<\kappa$. Clearly there is $h_{3} \in H_{2}$ taking $\mathscr{S}_{W} \cup\left\{\sum_{\mu<\kappa} a_{\lambda \mu} u_{1 \mu}+\sum_{\mu<\kappa} b_{\lambda \mu} w_{\mu}+u_{\lambda}^{\prime}: \lambda<\kappa\right\}$ onto $\mathscr{B}_{W} \cup\left\{\sum_{\mu<\kappa} a_{\lambda \mu} u_{1 \mu}+u_{2 \lambda}: \lambda<\kappa\right\}$. Next, choose $h_{4} \in H_{1}$ so that $u_{1 \lambda} h_{4}=w_{\lambda}$ and $w_{\lambda} h_{4}=u_{1 \lambda}$. Then

$$
\mathscr{B}^{\prime} h_{1} h_{2} h_{3} h_{4}=\mathscr{B}_{1} \cup \mathscr{B}_{W} \cup\left\{\sum_{\mu<\kappa} a_{\lambda \mu} w_{\mu}+u_{2 \lambda}: \lambda<\kappa\right\} .
$$

Now there is $h_{5} \in H_{2}$ so that $\mathscr{B}^{\prime} h_{1} h_{2} h_{3} h_{4} h_{5}=\mathscr{B}$, as required.

The notations of filter and ideal on a set have natural vector space analogues. An ideal on $V$ is a family $\mathscr{I}$ of subspaces of $V$ such that

(i) $\{0\} \in \mathscr{I}$ and $V \notin \mathscr{I}$.

(ii) if $U \in \mathscr{I}$ and $W \leq U$ then $W \in \mathscr{I}$, 
(iii) if $U_{1}, U_{2} \in \mathscr{I}$ then $U_{1}+U_{2} \in \mathscr{I}$.

Similarly, a filter on $V$ is a family $\mathscr{F}$ of subspaces of $V$ such that

(i) $V \in \mathscr{F},\{0\} \notin \mathscr{F}$,

(ii) if $U \in \mathscr{F}$ and $U \leq W \leq V$ then $W \in \mathscr{F}$,

(iii) if $U_{1}, U_{2} \in \mathscr{F}$ then $U_{1} \cap U_{2} \in \mathscr{F}$.

Note that, by Zorn's lemma, every ideal (respectively filter) on $V$ is contained in a maximal ideal (respectively filter) on $V$. Note too that there is no natural duality between ideals and filters on $V$. If $\mathscr{S}$ is any family of subspaces of $V$ then

$$
G_{\{\mathscr{S}\}}:=\{g \in G: \text { for all } U \leq V, U \in \mathscr{S} \text { if and only if } U g \in \mathscr{S}\} .
$$

Note that $G$ has a natural action on the set of all maximal ideals on $V$, and on the set of all maximal filters on $V$. By the next theorem, this yields a large family of maximal subgroups of $G$.

THEOREM 2.3. If $\mathscr{S}$ is either a maximal ideal or a maximal filter on $V$ then $G$ acts 2-transitively on the set of all $G$-translates of $\mathscr{S}$, and hence $G_{\{\mathscr{S}\}}$ is a maximal subgroup of $G$.

Lemma 2.4. (i) Let $\mathscr{I}_{1}, \mathscr{J}_{2}$ be maximal ideals on $V$ such that for every moietous subspace $W$ of $V, W \in \mathscr{J}_{1}$ if and only if $W \in \mathscr{F}_{2}$. Then $\mathscr{I}_{1}=\mathscr{I}_{2}$.

(ii) The same as (i) holds with filters replacing ideals throughout.

(iii) Every maximal ideal or filter on $V$ contains a moietous subspace of $V$.

Proof. (i) It suffices to show that if $U \in \mathscr{J}_{1}$ and $\operatorname{Dim} U<\kappa$ then there is some moietous subspace $W$ of $V$ with $U \leq W$ and $W \in \mathscr{J}_{1}$. So let $W_{0}$ be moietous in $V$. If $W_{0} \notin \mathscr{I}_{1}$ then there is $W_{1} \in \mathscr{I}_{1}$ with $W_{0} \oplus W_{1}=V$. As $U, W_{1} \in \mathscr{I}_{1}, U+W_{1} \in \mathscr{J}_{1}$. Clearly $U+W_{1}$ is moietous in $V$.

(ii) This is similar to (i).

(iii) Let $\mathscr{I}$ be a maximal ideal on $V$, and let $W$ be a moietous subspace of $V$. If $W \notin \mathscr{I}$ then by maximality of $\mathcal{I}, W$ has a complement $U$ in $V$ with $U \in \mathscr{F}$. Since $U$ is moietous in $V$, this yields the result for ideals. The proof for filters is similar.

The following lemmas are analogues for vector spaces of results which are immediate for symmetric groups.

Lemma 2.5. Let $\mathscr{I}$ be an ideal on $V$, and put $G_{(\mathscr{F})}:=\bigcup\left\{G_{\{U\},(V / U)}\right.$ : $U \in \mathscr{I}\}$. Then $G_{(\mathscr{I})}$ is a normal subgroup of $G_{\{\mathscr{I}\}}$. 
Proof. Claim 1. If $U \in \mathscr{I}$ and $W \leq V$ with $U \oplus W=V$, then $G_{(W),\{U\}} \leq G_{\{\mathscr{I}\}}$.

Proof of ClAIM. Let $T \in \mathscr{I}$ and say $T$ has a basis $\left(t_{\lambda}: \lambda<\mu\right)$ with $t_{\lambda}=u_{\lambda}+w_{\lambda}$ where $u_{\lambda} \in U$ and $w_{\lambda} \in W$, for all $\lambda<\mu$. Now if $g \in$ $\hat{G}_{(W),\{U\}}$ then $t_{\lambda} g=u_{\lambda} g+w_{\lambda}$ for all $\lambda<\mu$. Since $T+U \in \mathcal{I}$, and $\left\langle w_{\lambda}: \lambda<\mu\right\rangle \leq T+U,\left\langle w_{\lambda}: \lambda<\mu\right\rangle \in \mathscr{I}$. Hence, as $u_{\lambda} g \in U$ for all $\lambda<\mu$, $\left\langle t_{\lambda} g: \lambda\langle\mu\rangle \leq U+\left\langle w_{\lambda}: \lambda\langle\mu\rangle\right.\right.$ so $T g \in \mathscr{F}$, as required.

Claim 2. In the notation of Claim 1, $G_{\{\mathscr{I}\},(U),(W / U)}$ is transitive on the set of complements of $U$ in $V$.

Proof of Claim 2. Suppose $U \oplus W_{1}=U \oplus W_{2}=V$, and let $W_{1}$ have basis $\left\{w_{\lambda}: \lambda<\mu\right\}$. Then $W_{2}$ has a basis $\left\{u_{\lambda}+w_{\lambda}: \lambda<\mu\right\}$ where all the $u_{\lambda}$ lie in $U$. Let $g$ be the element of $G$ fixing $U$ pointwise and mapping each $w_{\lambda}$ to $u_{\lambda}+w_{\lambda}$. Since $W_{1} g=W_{2}$, it suffices to show $g \in G_{\{\mathscr{S}\}}$. So suppose $T \in \mathscr{J}$. Then $T$ has a basis $\left(u_{\lambda}^{\prime}+\sum_{\nu<\mu} a_{\lambda \nu} w_{\nu}: \lambda<\mu, u_{\lambda}^{\prime} \in U, a_{\lambda \nu} \in F\right)$. Now clearly $T_{W}:=\left\langle\sum_{\nu<\mu} a_{\lambda \nu} w_{\nu}: \lambda<\mu, a_{\lambda \nu} \in F\right\rangle \in \mathscr{I}$, since it is a subspace of $U+T$. Also, $T g$ has a basis $\left(u_{\lambda}^{\prime}+\sum_{\nu<\mu} a_{\lambda \nu}\left(u_{\nu}+w_{\nu}\right): \lambda<\mu\right.$, $u_{\lambda}^{\prime} \in U, a_{\lambda \nu} \in F$ ). Hence $T g \leq U+T_{W}$, so as $U+T_{W} \in \mathscr{I}, T g \in \mathscr{I}$. A similar argument shows that if $R \in \mathscr{F}$ then $R g^{-1} \in \mathscr{I}$. Hence $g \in G_{\{\mathscr{I}\}}$ as required.

It follows easily from Claims 1 and 2 that for each $U \in \mathscr{I}, G_{\{U\},(V / U)} \leq$ $G_{\{\mathscr{I}\}}$. Thus, the lemma will follow if we can show that $G_{(\mathscr{F})}$ is a group. However, it is easily verified that if $U_{1}, U_{2} \in \mathscr{I}$ and $g_{1} \in G_{\left\{U_{1}\right\},\left(V / U_{1}\right)}$, $g_{2} \in G_{\left\{U_{2}\right\},\left(V / U_{2}\right)}$ then $g_{1} g_{2} \in G_{\left\{U_{1}+U_{2}\right\},\left(V /\left(U_{1}+U_{2}\right)\right)}$, and as $U_{1}+U_{2} \in \mathcal{F}$ this shows that $g_{1} g_{2} \in G_{(\mathscr{I})}$.

Lemma 2.6. Let $\mathscr{F}$ be a filter on $V$, and let $G_{(\mathscr{T})}:=\{g \in G: \operatorname{Fix}(g) \in$ $\mathscr{F}\}$. Then $G_{(\mathscr{F})}$ is a normal subgroup of $G_{\{\mathscr{F}\}}$.

Proof. This is immediate.

REMARK. Lemmas 2.5 and 2.6, together with Theorem 6.4 of [8] (also proved earlier in [9]), suggest the following question. If $\mathscr{S}$ is a maximal filter or maximal ideal on $V$, do we have $G_{(\mathscr{S})}=G_{\{\mathscr{S}\}}$ ?

LEMMA 2.7. Let $\mathscr{S}$ be a maximal filter (respectively maximal ideal) on $V$, and let $U$ be moietous subspace of $V$ lying in $\mathscr{S}$. Then there is $W \in \mathscr{S}$ such that $W$ is a moietous subspace of $U$ (respectively, $U$ is a moietous subspace of $W$, and $W$ is a moietous subspace of $V$ ). 
Proof. Let $\mathscr{S}$ be a filter. Choose a moietous subspace $X$ of $U$. If $X \notin \mathscr{S}$ then by maximality there is $Y \in \mathscr{S}$ with $X \cap Y=0$. Now choose $W$ to be any moietous subspace of $U$ containing $Y \cap U$. The proof for ideals is similar.

LeMMA 2.8. Let $\mathscr{S}$ be a maximal ideal or maximal filter on $V$. Then $G_{\{\mathscr{S}\}}$ is transitive on the set of moietous subspaces of $V$ which lie in $\mathscr{S}$.

Proof. Suppose that $\mathscr{S}$ is a maximal ideal. Let $U_{1}, U_{2}$ be moietous subspaces of $V$ lying in $\mathscr{S}$. If $U_{1}+U_{2}$ is not moietous in $V$, then both $U_{1}$ and $U_{2}$ are moietous in $U_{1}+U_{2}$; hence, there is $g \in G_{\left\{U_{1}+U_{2}\right\},\left(V /\left(U_{1}+U_{2}\right)\right)}$ with $U_{1} g=U_{2}$, and by Lemma 2.5 and the fact that $U_{1}+U_{2} \in \mathscr{S}, g \in G_{\{\mathscr{S g}\}}$, as required. If $U_{1}+U_{2}$ is moietous in $V$, then by Lemma 2.7 there is $W \in \mathscr{S}$ such that $U_{1}+U_{2}$ is moietous in $W$ and $W$ is moietous in $V$; hence there is $g \in G_{\{W\},(V / W)}$ with $U_{1} g=U_{2}$, and by Lemma 2.5 we have $g \in G_{\{\mathscr{S}\}}$, as required.

We omit the proof when $\mathscr{S}$ is a filter, since it is very similar (it uses Lemmas 2.6 and 2.7).

Proof of Theorem 2.3. Let $\mathscr{S}$ be a maximal ideal, and let $\Omega$ be the set of $G$-translates of $\mathscr{S}$. Let $\mathscr{I}_{1}, \mathscr{J}_{2}, \mathscr{F}_{1}, \mathscr{J}_{2} \in \Omega$ with $\mathscr{I}_{1} \neq \mathscr{J}_{2}$ and $\mathscr{J}_{1} \neq \mathscr{J}_{2}$. By Lemma 2.4(i) there is a moietous subspace $U_{1}$ of $V$ with $U_{1} \in$ $\mathscr{F}_{1} \mid \mathscr{F}_{2}$, and as $U_{1} \notin \mathscr{I}_{2}$ and by maximality of $\mathscr{S}, U_{1}$ has a complement $U_{2}$ in $V$ with $U_{2} \in \mathscr{F}_{2} \backslash \mathscr{J}_{1}$. Similarly, there are moietous subspaces $W_{1}, W_{2}$ of $V$ with $W_{1} \in \mathscr{J}_{1} \mathscr{F}_{2}, W_{2} \in \mathscr{Z}_{2} \backslash \mathscr{J}_{1}$, and $V=W_{1} \oplus W_{2}$. By Lemmas 2.5 and 2.8 there are $g_{1}, g_{2} \in G$ such that for all $i, j \in\{1,2\}, \mathcal{F}_{j} g_{j}=\mathscr{F}_{j}$ and $U_{i} g_{j}=W_{i}$ (here, 2.5 is used since we need that for any $W \in \mathscr{S}$, $G_{\{\mathscr{S}\},\{W\}}$ is transitive on the set of complements of $W$ in $\left.V\right)$. Let $g$ be the unique element of $G$ inducing $g_{1}$ on $U_{2}$ and $g_{2}$ on $U_{1}$. Now $g g_{1}^{-1} \in G_{\left\{U_{1}\right\},\left(V / U_{1}\right)} \leq G_{\left\{\mathcal{F}_{1}\right\}}$ (by Lemma 2.5), so $\mathscr{F}_{1} g=\mathscr{J}_{1}$. Similarly, $\mathscr{F}_{2} g=\mathscr{J}_{2}$.

The proof when $\mathscr{S}$ is a filter is very similar, and we omit it.

For the final part of Theorem 2.3 recall that a transitive permutation group $H$ on a set $X$ is primitive if and only if, for every $x \in X, H_{x}$ is maximal in $H$. Since 2-transitive permutation groups are primitive, this yields maximality of $G_{\{\mathscr{S}\}}$.

Question. Is the degree of transitivity in the actions of Theorem 2.3 greater than two?

One would expect Theorem 2.3 to yield a large number of non-conjugate maximal subgroups. This is certainly true if the field is not too large. We call 
an ideal (respectively filter) uniform if it contains no subspace of codimension in $V$ (respectively dimension) less than $\kappa$.

LEMMA 2.9. There are at least $2^{2^{x}}$ uniform maximal ideals on $V$, and at least $2^{2^{k}}$ uniform maximal filters on $V$.

Proof. We prove the result for ideals, the proof for filters being similar. Let $\mathscr{S}$ be the set of subspaces of $V$ of dimension less than $\kappa$. Also let $\mathscr{B}=\left(v_{\lambda}: \lambda<\kappa\right)$ be an ordered basis of $V$. By a theorem of Pospisil (Jech [5; p. 256]) there are $2^{2^{x}}$ distinct uniform maximal ideals on $\mathscr{B}$, $\left(\mathscr{F}_{\lambda}: \lambda<2^{2^{\kappa}}\right)$ say (here, "ideal" just means ideal of subsets of a set, and an ideal $\mathscr{J}$ on $\mathscr{B}$ is uniform if, for every $A \in \mathscr{J},|\mathscr{B} \backslash A|=\kappa)$. For each $\lambda<2^{2^{K}}$ let $\mathscr{I}_{\lambda}$ be the ideal on $V$ generated by

$$
\mathscr{S} \cup\left\{\langle A\rangle: A \in \mathscr{J}_{\lambda}\right\}
$$

(note that each $\mathscr{I}_{\lambda}$ is an ideal, that is, does not contain $V$ ). By Zorn's lemma each $\mathscr{F}_{\lambda}$ is contained in a maximal ideal $\mathscr{I}_{\lambda}^{*}$ on $V$, and, as $\mathscr{S} \subseteq \mathscr{F}_{\lambda}, \mathscr{F}_{\lambda}^{*}$ is uniform. Clearly the ideals $\mathscr{F}_{\lambda}^{*}$ are all distinct, since the $\mathscr{F}_{\lambda}$ are distinct.

LeMma 2.10. Distinct maximal ideals on $V$ have distinct stabilisers, and distinct maximal filters on $V$ have distinct stabilisers.

Proof. We prove the result for ideals, the proof for filters being similar. It suffices by Lemma 2.4 to show that if $\mathscr{I}$ is a maximal ideal then $\mathscr{I}$ contains every moietous subspace $U$ of $V$ for which $G_{\{\mathscr{I}\}} \geq G_{\{U\},(V / U)}$. So suppose $W \notin \mathscr{I}$ where $W$ is moietous in $V$ and $G_{\{W\},(V / W)} \leq G_{\{\mathscr{J}\}}$. As $W \notin \mathscr{I}$ and $\mathscr{I}$ is maximal, there is $U \in \mathscr{I}$ with $U \oplus W=V$. Furthermore, by Lemma 2.7 there is $X \in \mathscr{F}$ such that $U$ is moietous in $X$ and $X$ is moietous in $V$. Proposition 2.2 now shows that $G_{\{\mathscr{F}\}}=G$, a contradiction.

Corollary 2.11. Provided the cardinal $\left(\kappa \cdot|F|^{\kappa}\right)$ is less than $2^{2^{\kappa}}$, G has $2^{2^{\kappa}}$ distinct non-conjugate maximal subgroups.

Proof. First, note that since $|G|$ is equal to the number of ordered bases of $V,|G|=\left(\kappa \cdot|F|^{\kappa}\right)$. The result now follows from Theorem 2.3 and Lemmas 2.9 and 2.10 .

QUESTION. If the assumption in Corollary 2.11 fails, how many maximal subgroups does $G$ have up to conjugacy? In particular, how many maximal ideals and maximal filters are there? 
REMARK. In the above material on filters and ideals, we have essentially had to give the same proof twice, once for filters and once for ideals. It would be interesting to know if there is any way to make these proofs uniform.

We now obtain some further maximal subgroups of $G$. As the proofs are similar to earlier arguments, we omit some details. If $\lambda \leq \kappa$ is a cardinal and $U, W$ are subspaces of $V$ of dimension $\lambda$, then we write $U=_{\lambda} W$ if $\operatorname{Dim}((U+W) / U)+\operatorname{Dim}((U+W) / W)<\lambda$. Note that $=_{\lambda}$ is an equivalence relation (to see transitivity, note that if $X={ }_{\lambda} Y={ }_{\lambda} Z$ then $\operatorname{Dim}((X+Y+$ $Z) / Y)<\lambda$ so $\operatorname{Dim}((X+Y+Z) /(X+Y))<\lambda$; hence, as $\operatorname{Dim}((X+Y) / X)<$ $\lambda, \operatorname{Dim}((X+Y+Z) / X)<\lambda$, so $\operatorname{Dim}((X+Z) / X)<\lambda$ and a similar argument gives $\operatorname{Dim}((X+Z) / Z)<\lambda)$.

TheOREM 2.12. Let $U \leq V$ with $\operatorname{Dim} U=\lambda \leq \kappa$ and $\operatorname{Dim}(V / U)=\kappa$. Let $H:=\left\{g \in G: U g={ }_{\lambda} U\right\}$. Then $H$ is a group, and is maximal in $G$.

REMARK. We may regard $H$ as the almost stabiliser of the subspace $U$. Note that $H \geq G_{\{U\}} \cdot G_{\lambda}^{*}$, with equality if and only if $\lambda>\aleph_{0}$.

Proof. We omit the proof that $H$ is a group. Suppose first that $\lambda<$ $\kappa$. Let $g \in G \backslash H$. By replacing $g$ by $g^{-1}$ if necessary, we may suppose that $\operatorname{Dim}((U+U g) / U)=\lambda$, so $U$ is moietous in $U+U g$. It is easily seen that there is $k \in\langle H, g\rangle$ such that $U \cap U k=0$. Choose moietous subspaces $V_{1}, V_{2}, W$ of $V$ such that $V=\left(U \oplus V_{1}\right) \oplus W \oplus\left(U k \oplus V_{2}\right)$. Then $\langle H, g\rangle$ contains both $G_{\left\langle U \oplus V_{1}\right),\left\{U k \oplus V_{2} \oplus W\right\}}$ and $G_{\left(U k \oplus V_{2}\right),\left\{U \oplus V_{1} \oplus W\right\}}$. Hence, by Proposition 2.2, $G=\langle H, g\rangle$, as required.

Suppose now that $\lambda=\kappa$. Then we may assume (by adjusting by elements of $H$ if necessary) that $U$ is moietous in $U g$, or $U g$ is moietous in $U$, or $U \cap U g$ is moietous in each of $U, U g$, or $U$ and $U g$ generate their direct sum which is moietous in $V$, or $U \oplus U g=V$. The first three possibilities can be reduced to the fourth, and in the fourth, much as in the last paragraph, an application of Proposition 2.2 shows that $\langle H, g\rangle=G$. So suppose that the fifth case holds, that is $U \oplus U g=V$. Let $U g=W_{1} \oplus W_{2}$ be a decomposition into moietous subspaces, let $\left(u_{\mu}: \mu<\kappa\right),\left(w_{\mu}: \mu<\kappa\right)$ be ordered bases of $U, W_{2}$ respectively, and let $W_{3}:=\left\langle u_{\mu}+w_{\mu}: \mu\langle\kappa\rangle\right.$. Clearly $\langle H, g\rangle \geq$ $G_{(U),\left\{W_{1}+W_{2}\right\}}$. Also, there is $h \in H$ with $U g h=W_{1} \oplus W_{3}$; hence, as $W_{3} \oplus$ $\left(W_{1} \oplus W_{2}\right)=V$ and $H$ is transitive on the set of complements of $U$, there is $k \in\langle H, g\rangle$ with $U k=W_{2}$. It follows that $\langle H, g\rangle \geq G_{\left(W_{2}\right),\left\{U+W_{1}\right\}}$. Proposition 2.2 applied to $U, W_{1}, W_{2}$ now gives that $\langle H, g\rangle=G$.

REMARK. It is very easily checked that the stabiliser in $G$ of any finitedimensional subspace of $V$ is maximal in $G$. From Proposition 2.2, the same holds for subspaces of $V$ of finite codimension in $V$. More generally, 
if $U$ is a subspace of $V$ such that $\operatorname{Dim} U=\kappa$ and $\operatorname{Dim}(V / U)=\lambda$ where $\aleph_{0} \leq \lambda<\kappa$, then $\left\{g \in G: U g={ }_{\lambda} U\right\}$ is a maximal subgroup of $G$, again by Proposition 2.2 .

If $V=V_{1} \oplus \cdots \oplus V_{n} \quad(n<\omega)$ is a direct sum decomposition into moietous subspaces, then the almost stabiliser of the decomposition is

$$
H:=\left\{\begin{array}{c}
g \in G: \text { there is } \pi \in \operatorname{Sym}\{1, \ldots, n\} \text { such that for } \\
\text { all } i=1, \ldots, n, V_{i} g={ }_{\kappa} V_{i \pi}{ }^{-1}
\end{array}\right\} .
$$

Then $H$ is a group, and $H \geq G_{\kappa}^{*} \cdot\left(\mathrm{GL}\left(V_{1}\right) \times \cdots \times \mathrm{GL}\left(V_{n}\right)\right) \cdot S_{n}$, with equality if and only if $\kappa>\aleph_{0}$.

THEOREM 2.13. Let $V=V_{1} \oplus V_{2}$, where $V_{1}, V_{2}$ are moietous subspaces of $V$, and let $H$ be the almost stabiliser of the decomposition. Then $H$ is maximal in $G$.

Proof. Choose $g \in G \backslash H$ and put $K:=\langle H, g\rangle$. Also put $N:=\mathrm{GL}\left(V_{1}\right) \times$ $\mathrm{GL}\left(V_{2}\right)$.

Claim 1. There is $k \in K_{\left(V_{1}\right),\left(V / V_{1}\right)}$ such that $\operatorname{Dim}\left(\left(V_{2}+V_{2} k\right) / V_{2}\right)=\kappa$.

Subclaim 1. We may suppose there is no $h \in K$ such that $V_{1} h$ is a moietous subspace of $V_{1}$.

Proof of Subclaim. Suppose that there is such an $h$. Let $V_{1}=V_{1} h+Z$, and let $\left(z_{\lambda}: \lambda<\kappa\right)$ and $\left(v_{\lambda}: \lambda<\kappa\right)$ be ordered bases of $Z$ and $V_{2}$ respectively. Then $V_{2} h$ has a basis of the form $\left\{z_{\lambda}+s_{\lambda}, v_{\lambda}+t_{\lambda}: \lambda<\kappa\right\}$ where $s_{\lambda}, t_{\lambda} \in V_{1} h$. Now choose $k$ to be the linear transformation fixing $V_{1}$ pointwise and taking $v_{\lambda}+t_{\lambda}$ to $v_{\lambda}+t_{\lambda}+z_{\lambda}+s_{\lambda}$ for all $\lambda<\kappa$. Because $k$ fixes $V_{1} h$ and $V_{2} h$ setwise, $h k h^{-1} \in N$, so $k \in K$. Hence $k$ satisfies Claim 1.

From now on we make the assumption indicated in Subclaim 1. Now write $U_{1}:=V_{1} \cap V_{1} g, W_{1}:=V_{2} \cap V_{1} g, U_{2}:=V_{1} \cap V_{2} g, W_{2}:=V_{2} \cap V_{2} g$. There are cardinals $\mu, \nu<\kappa$ and linearly independent sets $\left\{x_{1 \lambda}: \lambda<\mu\right\},\left\{x_{2 \lambda}: \lambda<\right.$ $\nu\} \subseteq V_{1}$ and $\left\{y_{1 \lambda}: \lambda<\mu\right\},\left\{y_{2 \lambda}: \lambda<\nu\right\} \subseteq V_{2}$ such that

$$
\begin{aligned}
& V_{1} g=U_{1} \oplus W_{1} \oplus\left\langle x_{1 \lambda}+y_{1 \lambda}: \lambda<\mu\right\rangle, \\
& V_{2} g=U_{2} \oplus W_{2} \oplus\left\langle x_{2 \lambda}+y_{2 \lambda}: \lambda<\nu\right\rangle .
\end{aligned}
$$

SubClaim 2. We may assume that either

$$
U_{1} \oplus\left\langle x_{1 \lambda}: \lambda<\mu\right\rangle={ }_{\kappa} V_{1},
$$

or

$$
W_{1} \oplus\left\langle y_{1 \lambda}: \lambda<\mu\right\rangle={ }_{\kappa} V_{2}
$$


Proof of Subclaim. Suppose that neither possibility in the subclaim holds. Clearly one of $\operatorname{Dim} U_{1}, \operatorname{Dim} W_{1}, \mu$ is equal to $\kappa$. Hence there is $f \in N$ with $V_{1} g f$ moietous in $V_{1} g$. It follows that $V_{1} g f g^{-1}$ is moietous in $V_{1}$, contrary to our assumption after Subclaim 1 .

Subclaim 3. We may assume that $\mu=\kappa$.

Proof of Subclaim. Assume for a contradiction that $\mu<\kappa$. Suppose first (by Subclaim 2) that $U_{1} \oplus\left\langle x_{1 \lambda}: \lambda\langle\kappa\rangle={ }_{\kappa} V_{1}\right.$. Then either

(i) $\operatorname{Dim} W_{1}<\kappa$, and $V_{1} g={ }_{\kappa} V_{1}$, or $V_{1}$.

(ii) $\operatorname{Dim} W_{1}=\kappa$, and $V_{1} g$ contains a moietous subspace $X$ with $X={ }_{\kappa}$

In case (i) there is $f \in K$ such that $V_{1} g f=V_{1}$ and $\operatorname{Dim}\left(\left(V_{2} g f+\right.\right.$ $\left.\left.V_{2}\right) / V_{2}\right)=\kappa$. It follows that for some $n \in N$, the element $k:=g f n$ satisfies Claim 1. Case (ii) cannot hold, since it would contradict our assumption after Subclaim 1 (recall that $H$ contains the almost stabiliser of the decomposition $V_{1} \oplus V_{2}$ ). If the second possibility in Subclaim 2 holds, then a similar argument (using an involution which interchanges $V_{1}$ and $V_{2}$ ) again justifies the assumption that $\mu=\kappa$.

SubCLAim 4. We may assume that $\nu=\kappa$.

Proof of Subclaim. This is virtually the same as the proof of Subclaim 3 (with the obvious adaptation of Subclaim 2).

By Subclaims 3 and 4, from now on we can assume that $\mu=\nu=\kappa$.

Subclaim 5. We may assume $\operatorname{Dim} U_{1}=\operatorname{Dim} U_{2}=\kappa$.

Proof of Subclaim. It is possible to find $f \in N$ such that there are disjoint $\nu_{1}, \nu_{2} \subseteq \kappa$, each of size $\kappa$, with

$$
\left(x_{1 \lambda}+y_{1 \lambda}\right) f= \begin{cases}x_{1 \lambda}+y_{1 \lambda} & \text { if } \lambda \in \nu_{1}, \\ x_{2 \lambda}+y_{2 \lambda} & \text { if } \lambda \in \nu_{2} .\end{cases}
$$

Clearly $V_{1} g f \cap V_{1} g$ and $V_{1} g f \cap V_{2} g$ both have dimension $\kappa$. Hence $V_{1} g f g^{-1}$ intersects both $V_{1}$ and $V_{2}$ in subspaces of dimension $\kappa$. To obtain the subclaim, now replace $g$ by $g f g^{-1}$.

Now, to prove Claim 1 , let $U_{1}, U_{2}$ have bases $\left(u_{1 \lambda}: \lambda<\kappa\right),\left(u_{2 \lambda}: \lambda<\kappa\right)$. Choose $h \in N$ to fix $V_{2}, U_{1}$ and $V_{1} /\left(U_{1}+U_{2}\right)$ pointwise and to map $u_{2 \lambda}$ to $u_{2 \lambda}+u_{1 \lambda}$ for all $\lambda<\kappa$. Then $h$ fixes $V_{1} g$ setwise, and $\operatorname{Dim}\left(\left(V_{2} g+\right.\right.$ $\left.\left.V_{2} g h\right) / V_{2} g\right)=\kappa$. There is $n \in g^{-1} N g$ such that $h n$ fixes $V_{1} g$ and $V / V_{1} g$ pointwise and $\operatorname{Dim}\left(\left(V_{2} g+V_{2} g h n\right) / V_{2} g\right)=\kappa$. Now $k:=g h n g^{-1}$ satisfies the claim.

Claim 2. $K \geq G_{\left\{V_{1}\right\}}$. 
Proof of Claim. We argue as in the proof of [3, Proposition 2.3]. Note that $G_{\left\{V_{1}\right\}}$ is the semidirect product of $M:=G_{\left(V_{1}\right),\left(V / V_{1}\right)}$ by $N$, and that, as an $F N$-module, $M$ is naturally isomorphic to $\operatorname{Hom}\left(V_{2}, V_{1}\right)$ (here, if $\left(g_{1}, g_{2}\right) \in N$ and $\phi \in \operatorname{Hom}\left(V_{2}, V_{1}\right)$ then $\phi\left(g_{1}, g_{2}\right)$ sends $v_{2} \in V_{2}$ to $\left(\phi\left(v_{2} g_{2}^{-1}\right) g_{1}\right.$. Since $N \leq K$ and $k \in M \cap K$, we must show that any $F N$-invariant submodule $A$ of $\operatorname{Hom}\left(V_{2}, V_{1}\right)$ containing the element corresponding to $k$ is $\operatorname{Hom}\left(V_{2}, V_{1}\right)$. As $\operatorname{Dim} V_{1}=\operatorname{Dim} V_{2}$ we may identify them, and as in [3], $A$ becomes a 2-sided ideal in $\operatorname{Hom}\left(V_{2}, V_{2}\right)$. Such ideals are classified in [6] (see also [4, p. 258]), and since $A$ contains an element corresponding to $k$ (that is, an endomorphism of $V_{2}$ with infinite dimensional image), we have $A=\operatorname{Hom}\left(V_{2}, V_{2}\right)$, which proves the claim.

Given the claim it follows that $K \geq G_{\left\{V_{2}\right\}}$. Now by Theorem 2.12, $K=G$, as required.

Theorems 2.12 and 2.13 suggest that many of the maximal subgroups of the finite dimensional general linear groups may have infinite dimensional analogues. It would be interesting to investigate whether the almost stabilisers of other finite direct sum decompositions or of tensor decompositions, or the almost stabiliser of a symplectic form, if appropriately defined, ever give maximal subgroups. Note that if $\kappa=\omega$ and $V$ is endowed with a symplectic form $($,$) , then the corresponding symplectic group \operatorname{Sp}(V, F)$ also fixes the set $\mathscr{S}$ of all hyperplanes of $V$ which are of the form $v^{\perp}:=\{w \in V$ : $(v, w) \in 0\}$ for some $v \in V$. Let $\left\{e_{i}, f_{i}: i<\omega\right\}$ be a standard basis of $V$ with respect to $($,$) (so for all i, j<\omega,\left(e_{i}, e_{j}\right)=\left(f_{i}, f_{j}\right)=0$ and $\left.\left(e_{i}, f_{j}\right)=\delta_{i j}\right)$. Then $\mathscr{S}$ is the set of all those hyperplanes of $V$ which contain all but finitely many elements of $\left\{e_{i}, f_{i}: i<\omega\right\}$. It follows easily that the full stabiliser $H$ in $G$ of $\mathscr{S}$ induces the general linear group on each finite dimensional subspace of $V$, so properly contains $\operatorname{Sp}(V, F)$. Hence, under any reasonable definition, the almost stabiliser of the form $($,$) ought$ to contain $H$. (These observations were suggested by a conversation with Tim Penttila, and were made independently by Jon Hall.) There are other questions, such as the following, about large proper subgroups of $G$.

QUESTION. Is there a proper subgroup $H$ of $G$ such that for each moietous subspace $U$ of $V, H^{U}=\mathrm{GL}(U)$ ?

\section{Further remarks on $G$}

We here give for $G$ some further analogues of results proved in [8] for infinite symmetric group. Recall that if $N, M$ are groups, $N \unlhd M$ and 
$H \leq M$, then $H$ is a supplement for $N$ in $M$ if $N H=M$. In [8] (and also earlier, using $G C H$, in [11]) supplements of the normal subgroups of $S$ (the symmetric group on a set of size $\kappa$ ) are classified, and this sheds some light on the maximal subgroups of $S$ (see [8, Observation 6.1]). Also, in [8] we define the cofinality of an uncountable group $H$ to be the smallest order type of a chain of proper subgroups of $H$ with union $H$ and it is shown that the cofinality of $S$ is greater than $\kappa$. This is used in the classification of supplements of the normal subgroups of $S$. Here we obtain an analogous result on the cofinality, but do not have a similar classification.

THEOREM 3.1. The group $G$ has cofinality greater than $\kappa$.

We use the following lemma.

LEMMA 3.2. Let $H<G$ and suppose there is a decomposition $V=U \oplus W$ into moietous subspaces such that $H_{\{U\},\{W\}}$ induces $\mathrm{GL}(U)$ on $U$. Then

(i) there are $g, h \in G$ such that $\langle H, g, h\rangle=G$,

(ii) $H$ is contained in a maximal subgroup of $G$.

Proof. (i) Choose $g \in G$ fixing $W$ pointwise, $U$ setwise, and, in its action on $U$, having fixed point space on $U$ of codimension $\kappa$ in $U$. Then by the results on normal subgroups of $G$ given in the introduction $\langle g\rangle$ has normal closure $G_{\{U\},(W)}$ in $H_{\{U\},(W)}$, so $\langle H, g\rangle$ contains $G_{\{U\},(W)}$. Next, let $U=U_{1} \oplus U_{2}$ be a decomposition into moietous subspaces and choose $h \in G$ with $U h=U_{1} \oplus W, W h=U_{2}$. Then $\left\langle G_{(W),\left\{U_{1}+U_{2}\right\}}, G_{\left(U_{2}\right),\left\{U_{1}+W\right\}}\right\rangle \leq$ $\langle H, g, h\rangle$, so by Proposition 2.2 we have $\langle H, g, h\rangle=G$.

(ii) This follows from (i) by Zorn's lemma, applied to the collection of all proper subgroups of $G$ which contain $H$ but omit at least one of $g, h$ (cf. [8, Lemma 6.9]).

Proof of Theorem 3.1. Suppose there is a chain $\left(H_{\lambda}: \lambda<\kappa\right)$ of proper subgroups of $G$ with union $G$. Choose a direct sum decomposition $V=$ $\bigoplus\left(V_{\lambda}: \lambda<\kappa\right)$ into moietous subspaces. For each $\lambda$, there is $g_{\lambda} \in \operatorname{GL}\left(V_{\lambda}\right)$ not induced by $H_{\lambda\left\{V_{\lambda}\right\},\left\{\bigoplus\left(V_{\mu}: \mu<\kappa, \mu \neq \lambda\right)\right\}}$ (for if this were false for some $\lambda$, then by Lemma 3.2 there would be $g, h \in G$ such that $\left\langle G_{\lambda}, g, h\right\rangle=G$; for some $\mu<\kappa$, we would have $g, h \in G_{\mu}$, and if $\nu=\operatorname{Max}\{\lambda, \mu\}$ then $G_{\nu}=G$, a contradiction). There is a unique $g \in G$ coinciding with $g_{\lambda}$ on $V_{\lambda}$ for each $\lambda$, and clearly $g \in G \backslash \bigcup\left(H_{\lambda}: \lambda<\kappa\right)$, a contradiction.

The next corollary was pointed out by Wilfrid Hodges. 
Corollary 3.3. Let $E$ be the ring of $F$-endomorphisms of $V$. Then $E$ is not the union of $a \kappa$-chain of proper subrings.

Proof. Suppose $E$ is the union of a chain of subrings $\left(E_{\lambda}: \lambda<\kappa\right)$. Since $G$ is a subgroup of the group of units of $E, G=\bigcup\left(G \cap E_{\lambda}: \lambda<\kappa\right)$. Hence, by Theorem 3.1, $G \leq E_{\lambda}$ for some $\lambda<\kappa$. However, by Zelinsky [12] each element of $E$ is a sum of two elements of $G$, so $E=E_{\lambda}$, as required.

Theorem 3.1 gives a little information about supplements of the normal subgroups $G_{\lambda}$. First, note the following lemma.

LEMMA 3.4. If $\kappa$ is regular then $G_{\kappa}$ has cofinality at most $\kappa$.

Proof. Let $\left(v_{\lambda}: \lambda<\kappa\right)$ be an ordered basis of $V$, and for each $\lambda<\kappa$ let $U_{\lambda}:=\left\langle v_{\mu}: \mu\langle\kappa\rangle\right.$ and $H_{\lambda}:=G_{\left\{U_{\lambda}\right\},\left(V / U_{\lambda}\right)}$. Then $H_{\lambda} \leq G_{\lambda}$ for all $\lambda<\kappa$, for if $h \in H_{\lambda}$, then $\operatorname{Im}(h-1) \leq U_{\lambda}$, so $\operatorname{Dim}(\operatorname{Im}(h-1))<\kappa$. Clearly also $H_{\lambda} \neq G_{\kappa}$ for all $\lambda<\kappa$. Finally, $\bigcup\left(H_{\lambda}: \lambda<\kappa\right)=G_{\kappa}$, for if $h \in G_{\kappa}$ then $\operatorname{Dim}(\operatorname{Im}(h-1))<\kappa$, so by the regularity of $\kappa, \operatorname{Im}(h-1) \leq U_{\lambda}$ for some $\lambda<\kappa$.

Question. Does Lemma 3.4 hold if $\kappa$ is singular?

QUESTION. Does $G_{\kappa}$ every have cofinality less than $\kappa$ ?

Now suppose that $K$ is a supplement for $G_{\kappa}$, that is, $K \leq G$ and $G_{\kappa} \cdot K=$ $G$. Then $\left(\left\langle H_{\lambda}, K\right\rangle: \lambda<\kappa\right)$ is a chain of length $\kappa$ of subgroups of $G$ with union $G$. Hence, by Theorem 3.1, there is $\lambda<\kappa$ such that $\left\langle H_{\lambda}, K\right\rangle=G$.

We conclude with an observation which must be well-known. We omit its proof, since it is routine.

Lemma 3.5. Let $E:=\operatorname{End}_{F}(V)$. Then there is an inclusion-preserving bijection $\phi$ between the set of filters of subspaces of $V$ and the set of proper right ideals of $E$, given by the rule: if $\mathscr{F}$ is a filter on $V$ then $\phi(\mathscr{F}):=\{x \in$ $E: \operatorname{Ker}(x) \in \mathscr{F}\}$.

\section{References}

[1] M. Brazil, J. A. Covington, T. Penttila, C. E. Praeger, A. Woods, 'Maximal subgroups of infinite symmetric groups', preprint.

[2] J. Dieudonné, 'Les determinants sur un corps non commutatif', Bull. Soc. Math. France 71 (1943), 27-45. 
[14] Maximal subgroups of infinite dimensional general linear groups

[3] D. M. Evans, 'Subgroups of small index infinite general linear groups', Bull. London Math. Soc. 18 (1986), 587-590.

[4] N. Jacobson, Lectures in abstract algebra, II Linear algebra (Van Nostrand, Toronto, 1953).

[5] T. Jech, Set theory (Academic Press, New York, 1978).

[6] R. E. Johnson, 'Equivalence rings', Duke Math. J. 15 (1948), 787-793.

[7] H. D. Macpherson, 'Large subgroups of infinite symmetric groups', preprint.

[8] H. D. Macpherson, P. M. Neumann, 'Subgroups of infinite symmetric groups', J. London Math. Soc. (2) 42 (1990), 64-84.

[9] F. Richmann, 'Maximal subgroups of infinite symmetric groups', Canad. Math. Bull. 10 (1967), 375-381.

[10] A. Rosenberg, 'The structure of the infinite general linear group', Ann. of Math. 68 (1958), 278-294.

[11] S. W. Semmes, 'Infinite symmetric groups, maximal subgroups and filters', Abstracts Amer. Math. Soc. 3 (1982), 38.

[12] D. Zelinsky, 'Every linear transformation is a sum of nonsingular ones', Proc. Amer. Math. Soc. 5 (1954), 627-630.

\section{School of Mathematical Sciences \\ Queen Mary and Westfield College \\ Mile End Road \\ London E1 4NS \\ England}

